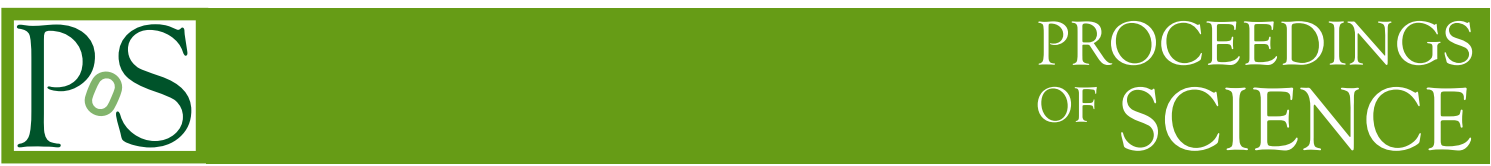

\title{
Pentaquark search with spatially non-trivial operators (II)
}

\author{
Ferenc Csikor ${ }^{a}$, Zoltán Fodor ${ }^{a b}$, Sándor D. Katz ${ }^{a b}$, Tamás G. Kovács ${ }^{c}$ and Bálint C. \\ Tóth*a \\ ${ }^{a}$ Institute for Theoretical Physics, Eötvös University, Hungary \\ ${ }^{b}$ Department of Physics, University of Wuppertal, Germany \\ ${ }^{c}$ Department of Theoretical Physics, University of Pécs, Hungary \\ E-mail: tothbalinteszofi.elte.hu
}

\begin{abstract}
We study spin $1 / 2$ isoscalar and isovector, even and odd parity candidates for the $\Theta^{+}(1540)$ pentaquark particle using large scale lattice QCD simulations. Two complications extracting the lowest state in a given parity channel are studied. Firstly, we summarize how the energy of the lowest state can be extracted separately in the two parity channels. Secondly, after summarizing the relevant group theoretical principles we discuss how spin $1 / 2$ pentaquark operators can be constructed.
\end{abstract}

XXIIIrd International Symposium on Lattice Field Theory

25-30 July 2005

Trinity College, Dublin, Ireland

\footnotetext{
*Speaker.
} 


\section{Introduction}

Recently several studies of non-trivial (exotic or excited) hadronic states appeared in the lattice literature. For extracting such states one typically needs several operators often with complicated spatial and internal structure and projection to states with the desired quantum numbers is not straightforward. Although all information is implicitly contained in the group theory literature, detailed discussions of these issues in lattice papers started to appear only recently. (See e.g. Refs. [1, 2, 3].) In the present paper we give an account of the operators and projections used for our pentaquark search [曰, 印.

For the proper analysis a large number of independent operators is needed, which span a large enough subspace containing the scattering states and a possible pentaquark state. The typical operators used in hadron spectroscopy contain quarks at only one lattice point with some Gaussian smearing. These operators have zero orbital angular momentum and give a very limited set of operators; e.g. the operator proposed by Jaffe and Wilczek [6, 7] cannot be implemented in this way. Therefore we decided to use spatially non-trivial operators containing quark fields at different lattice sites.

In Section 1 we summarize how the energy of the lowest state can be extracted separately in the two parity channels. In Section 3 we outline how a specific spin eigenstate can be projected out from a given lattice hadron operator. After summarizing the relevant group theoretical principles we discuss how our spin $1 / 2$ pentaquark operators were constructed.

\section{Parity projection}

The basic objects one can compute on the lattice are Euclidean correlators of the form $\left\langle\mathscr{O}_{\alpha}(x) \overline{\mathscr{O}}_{\beta}(0)\right\rangle$ corresponding to the amplitude of the process of creating a state at time zero with the operator $\overline{\mathscr{O}}_{\beta}(0)$, evolving it to a later time $x_{0}$ and annihilating it with $\mathscr{O}_{\alpha}(x)$.

There are two complications when one wants to extract the lowest state in a given parity channel. Firstly, simple baryonic operators usually couple to both parities, therefore one has to project out parity by hand. Secondly, the box has a finite time extent $T$ with (anti-)periodic boundary condition. Therefore, a single source at time zero is in fact mathematically equivalent to the sum of an infinite number of identical sources located at $t=0, \pm T, \pm 2 T \ldots$ Due to the exponential fall-off of correlations, only the two sources closest to the sink, i.e. at $t=0, T$ give appreciable contributions to the infinite sum. If we assume, as we shall always, that $0 \leq x_{0}<T$, then

$$
\sum_{n=-\infty}^{\infty}\left\langle\mathscr{O}_{\alpha}\left(\vec{x}, x_{0}\right) \overline{\mathscr{O}}_{\beta}(\overrightarrow{0}, n T)\right\rangle \approx\left\langle\mathscr{O}_{\alpha}\left(\vec{x}, x_{0}\right) \overline{\mathscr{O}}_{\beta}(\overrightarrow{0}, 0)\right\rangle+\varepsilon_{\mathrm{bc}}\left\langle\mathscr{O}_{\alpha}\left(\vec{x}, x_{0}\right) \overline{\mathscr{O}}_{\beta}(\overrightarrow{0}, T)\right\rangle
$$

where $\varepsilon_{\mathrm{bc}}$ is +1 for periodic and -1 for anti-periodic boundary condition in the time direction. The first term on the r.h.s. represents particles propagating from time 0 to $x_{0}$ while the second term represents antiparticles propagating from time $x_{0}$ to $T$. Thus even after projecting to a given parity channel the correlator has contributions not only from particles of that parity, but also from the antiparticles of the opposite parity. Therefore, an additional "projection" is needed to get rid of the latter.

Due to the transformation properties of $\mathscr{O}_{\alpha}$, the most general form the correlator can have is

$$
\left\langle\mathscr{O}_{\alpha}(x) \overline{\mathscr{O}}_{\beta}(0)\right\rangle=\left[f\left(x^{2}\right) x_{\mu} \gamma^{\mu}+g\left(x^{2}\right) \mathbf{1}\right]_{\alpha \beta} .
$$


After projection to the zero momentum sector the terms proportional to $\left\{\gamma_{i}\right\}_{i=1}^{3}$ vanish due to their antisymmetry and the resulting correlator is

$$
C_{\alpha \beta}\left(x_{0}\right)=\left[A\left(x_{0}\right) \gamma_{0}+B\left(x_{0}\right) \mathbf{1}\right]_{\alpha \beta}, \quad \text { where } \quad A\left(x_{0}\right)=\int \mathrm{d}^{3} x f\left(x^{2}\right) x_{0}, \quad B\left(x_{0}\right)=\int \mathrm{d}^{3} x g\left(x^{2}\right) .
$$

Parity projection. For ensuring that only states of a given parity propagate, it is enough to perform parity projection either at the sink or the source. Projection in eq. (2.3) at the sink yields

$$
\frac{1}{2}\left(\mathbf{1} \pm \eta \gamma_{0}\right)\left[A\left(x_{0}\right) \gamma_{0}+B\left(x_{0}\right) \mathbf{1}\right]= \pm \frac{\eta}{2}\left[A\left(x_{0}\right) \pm \eta B\left(x_{0}\right)\right] \mathbf{1}+\frac{1}{2}\left[A\left(x_{0}\right) \pm \eta B\left(x_{0}\right)\right] \gamma_{0},
$$

where $\eta= \pm 1$ is the internal parity of $\mathscr{O}$ and the plus and minus signs correspond to the positive and negative parity correlators, respectively. All the matrix elements of the parity projected correlator have the same functional dependence, $1 / 2(A \pm \eta B)$, on $x_{0}$, therefore the exponential fit to this function will yield the lowest state in the given parity channel.

"Particle" projection. Now the full correlator, including the term that "comes back" through the time boundary, has the form

$$
C\left(x_{0}\right)+\varepsilon_{\mathrm{bc}} C\left(x_{0}-T\right)=\left[A\left(x_{0}\right)-\varepsilon_{\mathrm{bc}} A\left(T-x_{0}\right)\right] \gamma_{0}+\left[B\left(x_{0}\right)+\varepsilon_{\mathrm{bc}} B\left(T-x_{0}\right)\right] \mathbf{1} .
$$

If we used the above prescription for parity projection, we would obtain

$$
\frac{1}{2}\left[A\left(x_{0}\right)+\eta B\left(x_{0}\right)\right]+\frac{\varepsilon_{\mathrm{bc}}}{2}\left[-A\left(T-x_{0}\right)+B\left(T-x_{0}\right)\right]
$$

for the parity projected correlator, which, due to an extra minus sign, does not have the simple functional form $f\left(x_{0}\right)+f\left(T-x_{0}\right)$ that could be fitted with a cosh. This extra sign, however, can be easily cancelled if we compute the $A$ and the $B$ components in eq. (2.3) with opposite boundary conditions. In that case the parity projected correlator has the form

$$
\frac{1}{2}\left[A\left(x_{0}\right)+\eta B\left(x_{0}\right)\right]+\frac{1}{2}\left[A\left(T-x_{0}\right)+\eta B\left(T-x_{0}\right)\right] .
$$

If $1 / 2\left[A\left(x_{0}\right)+\eta B\left(x_{0}\right)\right]$ is a sum of exponentials corresponding to the energies of the states in the given channel, 2.7) is a sum of cosh's with the same exponents. This is the functional form we have to use for fitting when extracting masses.

\section{Projection to a spin eigenstate}

Due to the absence of full $S O(3)$ rotational symmetry it is not straightforward to assign spin to a lattice energy eigenstate. States on the lattice can be classified into irreducible representations of the cubic group $O$ or its double cover ${ }^{2} O$, not $S O(3)$ or $S U(2)$ as in the continuum. For the character tables of $O$ and ${ }^{2} O$, see e.g. [8].

Irreducible representations of $S U(2)$, when restricted to ${ }^{2} O$, usually do not remain irreducible. The spin $0,1 / 2,1$ and $3 / 2 S U(2)$ representations are the exceptions, these restricted to ${ }^{2} O$ are equivalent to the irreducible representations $A_{1}, G_{1}, T_{1}$ and $H$, respectively. Also any state belonging to an irreducible representation of ${ }^{2} O$ has components belonging to several different spin representations of $S U(2)$. For instance a state in $G_{1}$ has components of spin $1 / 2,7 / 2,9 / 2, \ldots$. 
This means e.g. that if on the lattice we find the lowest energy state in the $G_{1}$ representation of ${ }^{2} \mathrm{O}$, we can identify that with a spin $1 / 2$ state in the continuum, provided all the higher spin states contributing to $G_{1}$, i.e. $s=7 / 2,9 / 2, \ldots$ can be assumed to have much higher energy. In this sense, for practical purposes, the lowest few representations of $S U(2)$ or ${ }^{2} O$ can be identified as follows:

$$
0 \leftrightarrow A_{1}, \quad 1 / 2 \leftrightarrow G_{1}, \quad 1 \leftrightarrow T_{1}, \quad 3 / 2 \leftrightarrow H .
$$

The task we have at hand is thus to construct states belonging to specific representations of the cubic group ${ }^{2} O$. This can be most easily done using the technique of projection operators. Let $G$ be a finite group, $D_{i j}^{(r)}(g)$ be the matrix elements of its irreducible representation $r$ of dimension $d_{r}$. Let the transformations $T(g)$ form an arbitrary (not necessarily irreducible) unitary representation of $G$. We would like to project a specific irreducible representation $r$ out of the carrier space of the $T(g)$ 's. Let us define the transformations

$$
P_{i j}^{(r)}=\frac{d_{r}}{|G|} \sum_{g \in G} D_{i j}^{(r) \star}(g) T(g) .
$$

If $|\psi\rangle$ is any vector belonging to the carrier space of $T(g)$ 's then for a fixed $j$ the $d_{r}$ vectors

$$
\left|\phi_{i}\right\rangle=P_{i j}^{(r)}|\psi\rangle, \quad i=1, \ldots, d_{r}
$$

either transform as basis vectors of the irreducible representation $r$ or they are all zero [9]. Equations (3.2) and (3.3) can be exploited to project out different representations of ${ }^{2} O$ from a given state and its rotated copies on the lattice.

In particular, we would like to construct pentaquark states belonging to $G_{1}$, which corresponds to spin $1 / 2$. In general the five-particle wave function could be any function of the locations of the five quarks. However, since the correlation functions are built up from quark propagators, we have to restrict ourselves to wave functions, which are products of the individual quark wave functions:

$$
O\left(x_{1}, x_{2}, x_{3}, x_{4}, x_{5}\right)=q_{1}\left(x_{1}\right) q_{2}\left(x_{2}\right) q_{3}\left(x_{3}\right) q_{4}\left(x_{4}\right) q_{5}\left(x_{5}\right) .
$$

Here, for simplicity we omitted the color and Dirac-structure. These are the elementary operators for which the correlators can be computed by single Dirac-matrix inversions. A general five-quark operator can be written as a linear combination of such elementary operators.

Although more complicated cases can also be considered, here we restrict ourselves to the one where the spin indices of all the quarks but one have been contracted to be scalars and the total spin of the pentaquark arises by combining the spin $1 / 2\left(G_{1}\right)$ of the remaining quark with the orbital angular momentum of all the constituents. Therefore we have to project $G_{1}$ out of $G_{1} \otimes s$, where $s$ is a representation of the cubic group $O\left(\right.$ not $^{2} O$ !), corresponding to the orbital part.

In practice $s$ depends on the spatial arrangement of quark sources and this can be exploited to make things as simple as possible. Eq. (3.2) implies that, in general, projection to a specific spin involves as many terms as the number of elements of the group ${ }^{2} O$, i.e. 48 . The situation, however, is much better if the projection formula (3.3) is applied to a state with an orbital part having some degree of symmetry under cubic rotations. For the quark wave-functions we use Gaussians centered on points of the $z$ axis only. Operators based on such wave-functions have axial symmetry and therefore the spin-projection requires at most five extra operators. Let

$$
q_{i}\left(d_{i}, r_{i}, x_{i}\right)=\exp \left(-\frac{\left(x_{i}-d_{i} \cdot \hat{z}\right)^{2}}{r_{i}^{2}}\right)
$$


where $\hat{z}$ is the unit vector along the $z$ axis. We will usually omit the $x_{i}$ argument.

The simplest case is when the five quark sources all have complete rotational symmetry, i.e. the orbital part is trivially $s=A_{1}$. Then all the rotated copies of the quark sources are identical, the sum in eq. (3.2) can be explicitly computed and the projection reduces to projection to spin up or spin down. The decomposition here is $A_{1} \otimes G_{1}=G_{1}$. We used two operators of this type.

$$
\begin{aligned}
\mathscr{O}_{1} & =\varepsilon^{a b c}\left[u_{a}^{T}(0,4) C \gamma_{5} d_{b}(0,4)\right]\left\{u_{c}(0,4) \bar{s}_{e}(0,4) \gamma_{5} d_{e}(0,4)+(u \leftrightarrow d)\right\} \\
\mathscr{O}_{2} & =\varepsilon^{a b c} \varepsilon^{a d e} \varepsilon^{b g h}\left[u_{d}^{T}(0,4) C \gamma_{5} d_{e}(0,4)\right]\left[u_{g}^{T}(0,4) C d_{h}(0,4)\right] C \bar{s}_{c}^{T}(0,4)
\end{aligned}
$$

The first operator has color index contractions corresponding to an $N-K$ state [10], while $\mathscr{O}_{2}$ has a diquark-diquark-antiquark type color structure [11].

To explore the possibility of non-zero orbital angular momentum we have to consider less symmetric quark sources. One possibility is to put the nucleon at the origin with a rotationally symmetric wave function and place the kaon along a coordinate axis (say $z$ ) keeping the arrangement cylindrically symmetric with respect to the $z$ axis:

$$
\varepsilon^{a b c}\left[u_{a}^{T}(0,4) C \gamma_{5} d_{b}(0,4)\right]\left\{u_{c}(0,4) \bar{s}_{e}\left(N_{s} / 2,4\right) \gamma_{5} d_{e}\left(N_{s} / 2,4\right)+(u \leftrightarrow d)\right\} .
$$

The relative displacement of the nucleon and kaon is half of the spatial lattice size, so this operator is spatially symmetric. Let us call such a state $|z\rangle$. It is easy to see that the rotated copies of this state span a three dimensional space carrying the reducible representation $A_{1} \oplus E$ of $O$. A possible set of basis states is given by the kaon displaced along the three coordinate axes: $|x\rangle,|y\rangle$ and $|z\rangle$. This arrangement corresponds to projecting out the spin $1 / 2\left(G_{1}\right)$ component from the decomposition

$$
G_{1} \otimes\left(A_{1} \oplus E\right)=G_{1} \otimes A_{1} \oplus G_{1} \otimes E=G_{1} \oplus H .
$$

Let us choose $|\psi\rangle=|\uparrow\rangle \otimes|z\rangle$ and compute $P_{11}^{\left(G_{1}\right)}|\psi\rangle$. The transformations $T(g)$ appearing in eq. (3.2) are direct products of $G_{1}$ transformations acting on the quark spin and transformations acting on the orbital part. Performing the projection

$$
P_{11}^{\left(G_{1}\right)}[|\uparrow\rangle \otimes|z\rangle]=|\uparrow\rangle \otimes(|x\rangle+|y\rangle+|z\rangle)
$$

we obtain our third operator

$$
\mathscr{O}_{3}=P^{\left(G_{1}\right)}\left[\varepsilon^{a b c}\left[u_{a}^{T}(0,4) C \gamma_{5} d_{b}(0,4)\right]\left\{u_{c}(0,4) \bar{s}_{e}\left(N_{s} / 2,4\right) \gamma_{5} d_{e}\left(N_{s} / 2,4\right)+(u \leftrightarrow d)\right\}\right],
$$

a shifted $N-K$ scattering operator with spin projection.

Another possibility is to put the antiquark at the origin with a rotationally symmetric wave function and displace two pairs of $(u d)$ quarks along the $z$ axis. Inspired by the Jaffe-Wilczek diquark-diquark-antiquark picture [6, 7], in anticipation of orbital angular momentum 1, we construct this state to be antisymmetric with respect to space reflection:

$$
\varepsilon^{a b c} \varepsilon^{a d e} \varepsilon^{b g h}\left[u_{d}^{T}(1,2) C \gamma_{5} d_{e}(1,2)\right]\left[u_{g}^{T}(-1,2) C \gamma_{5} d_{h}(-1,2)\right] C \bar{s}_{c}^{T}(0,4) .
$$

Our other antisymmetric operator is constructed from a nucleon and a kaon placed at a distance of $N_{s} / 4$ from each other:

$$
\begin{aligned}
& \varepsilon^{a b c}\left[u_{a}^{T}(0,4) C \gamma_{5} d_{b}(0,4)\right]\left\{u _ { c } ( 0 , 4 ) \left[\bar{s}_{e}\left(N_{s} / 4,4\right) \gamma_{5} d_{e}\left(N_{s} / 4,4\right)\right.\right. \\
&\left.\left.-\bar{s}_{e}\left(-N_{s} / 4,4\right) \gamma_{5} d_{e}\left(-N_{s} / 4,4\right)\right]+(u \leftrightarrow d)\right\} .
\end{aligned}
$$


Let us call such an antisymmetric state $| \pm z\rangle$. The rotated copies of this state span the three dimensional representation $T_{1}$ of $O$ with basis vectors $| \pm x\rangle,| \pm y\rangle$ and $| \pm z\rangle$. Thus we have to project out the spin $1 / 2$ component from

$$
G_{1} \otimes T_{1}=G_{1} \oplus H
$$

The explicit form of the projection

$$
P_{11}^{\left(G_{1}\right)}[|\uparrow\rangle \otimes| \pm z\rangle]=|\downarrow\rangle \otimes[| \pm x\rangle+i \cdot| \pm y\rangle]+|\uparrow\rangle \otimes| \pm z\rangle,
$$

is identical to the spin $1 / 2$ part of the $S U(2)$ Clebsch-Gordan decomposition $1 / 2 \otimes 1=1 / 2 \oplus 3 / 2$. This way for our fourth and fifth operators we obtain

$$
\begin{aligned}
\mathscr{O}_{4}= & P^{\left(G_{1}\right)}\left[\varepsilon^{a b c} \varepsilon^{a d e} \varepsilon^{b g h}\left[u_{d}^{T}(1,2) C \gamma_{5} d_{e}(1,2)\right]\left[u_{g}^{T}(-1,2) C \gamma_{5} d_{h}(-1,2)\right] C \bar{s}_{c}^{T}(0,4)\right], \\
\mathscr{O}_{5}= & P^{\left(G_{1}\right)}\left[\varepsilon ^ { a b c } [ u _ { a } ^ { T } ( 0 , 4 ) C \gamma _ { 5 } d _ { b } ( 0 , 4 ) ] \left\{u _ { c } ( 0 , 4 ) \left[\bar{s}_{e}\left(N_{s} / 4,4\right) \gamma_{5} d_{e}\left(N_{s} / 4,4\right)\right.\right.\right. \\
& \left.\left.\left.-\bar{s}_{e}\left(-N_{s} / 4,4\right) \gamma_{5} d_{e}\left(-N_{s} / 4,4\right)\right]+(u \leftrightarrow d)\right\}\right]
\end{aligned}
$$

\section{References}

[1] S. Basak et. al., Group-theoretical construction of extended baryon operators in lattice QCD, hep-lat/0506029.

[2] Lattice Hadron Physics (LHPC) Collaboration, S. Basak et. al., Clebsch-Gordan construction of lattice interpolating fields for excited baryons, hep-lat/0508018.

[3] D. C. Moore and G. T. Fleming, Angular momentum on the lattice: The case of non-zero linear momentum, hep-lat/0507018.

[4] F. Csikor, Z. Fodor, S. D. Katz, T. G. Kovács and B. C. Tóth, Pentaquark search with spatially non-trivial operators (I), PoS(LAT2005)066 (2005).

[5] F. Csikor, Z. Fodor, S. D. Katz, T. G. Kovács and B. C. Tóth, A comprehensive search for the $\theta^{+}$ pentaquark on the lattice, hep-lat/0503012.

[6] R. L. Jaffe and F. Wilczek, Diquarks and exotic spectroscopy, Phys. Rev. Lett. 91 (2003) 232003 hep-ph/0307341].

[7] R. Jaffe and F. Wilczek, Systematics of exotic cascade decays, Phys. Rev. D69 (2004) 114017 [hep-ph/0312369].

[8] R. C. Johnson, Angular momentum on a lattice, Phys. Lett. B114 (1982) 147.

[9] A. O. Barut and R. Raczka, Theory of group representations and applications, . Singapore, Singapore: World Scientific (1986) 717p.

[10] F. Csikor, Z. Fodor, S. D. Katz and T. G. Kovács, Pentaquark hadrons from lattice QCD, JHEP 11 (2003) 070 hep-lat/0309090.

[11] S. Sasaki, Lattice study of exotic $S=+1$ baryon, Phys. Rev. Lett. 93 (2004) 152001 hep-lat/0310014. 